\title{
GESTÃO DE CUSTOS NO CENTRO CIRÚRGICO: IMPACTO FINANCEIRO E PERDA DE RECEITA
}

\author{
Cost management at the operating room: financial impact and loss of revenue
}

Gestión de costos en el quirófano: impacto financiero y pérdida de ingresos

Mayara Martins de Sousa' ${ }^{1}$, Rachel de Carvalho ${ }^{2 *}$ (D)

RESUMO: Objetivo: Elencar os materiais de maior impacto financeiro no centro cirúrgico e estimar a perda de receita que representaram para a instituição. Método: Pesquisa retrospectiva e quantitativa, com coleta em banco de dados e planilhas do centro cirúrgico e garantia da receita dos anos de 2015 e 2018 de uma instituição privada de extra porte de São Paulo. Resultados: Os materiais que causaram maior impacto financeiro foram: Sevorane ${ }^{\circledR}$, Plasma Lyte $^{\circledast}$, manta térmica, sensor de monitor bispectral, máscara de anestesia, caneta para bisturi, perneira Sequel ${ }^{\circledast}$ e placa para bisturi. Representaram perda financeira de R \$ 342.473,00 em 2015, quando ainda não havia a prática de auditoria in loco, comparada ao valor de R\$ 114.157,00, em 2018, após ação de conferências em sala operatória. Conclusão: Houve benefício financeiro alcançado com a prática da auditoria in loco, com declínio de 66,6\% na quantidade de materiais e medicamentos excluídos pela auditoria em razão da elevação da qualidade das anotações em prontuário.

Palavras-chave: Enfermagem de centro cirúrgico. Registros hospitalares. Custos e análise de custo. Organização e administração.

ABSTRACT: Objective: To list the materials of greatest financial impact in the operating room and to estimate the loss of revenue they represented for the institution. Method: Retrospective and quantitative research, with collection in the database and spreadsheets of the surgical center and revenue guarantee for the years 2015 and 2018 of a private and large institution in São Paulo. Results: The materials that had the greatest financial impact were: Sevorane ${ }^{\circledast}$, Plasma Lyte ${ }^{\circledast}$, thermal blanket, bispectral monitor sensor, anesthesia mask, eletric scalpel pen, Sequel ${ }^{\circledast}$ leg protector, and electrosurgical plate. They represented a financial loss of BRL 342,473.00 in 2015, when there was still no on-site audit practice, compared with the amount of BRL 114,157.00, in 2018, after implementing checking initiatives in the operating room. Conclusion: There was a financial benefit achieved with the practice of on-site audit, with a $66.6 \%$ decline in the amount of materials and medicines excluded by the audit due to the enhancement in the quality of the notes found in medical records.

Keywords: Operating room nursing. Hospital records. Costs and cost analysis. Organization and administration.

RESUMEN: Objetivo: Enumerar los materiales con mayor impacto financiero en el Centro Quirúrgico y estimar la pérdida de ingresos que representaron para la institución. Método: Investigación retrospectiva y cuantitativa, con recolección en la base de datos y hojas de cálculo del Centro Quirúrgico y garantía de los ingresos para los años 2015 y 2018, de una institución privada extra grande de São Paulo. Resultados: Los materiales que causaron mayor impacto económico fueron: Sevorane ${ }^{\circledR}$, Plasma Lyte ${ }^{\circledast}$, Manta térmica, Sensor monitor biespectral, Máscara de anestesia, Pluma para bisturí, Perneira Seque ${ }^{\oplus}$ y Placa para bisturí. Representaron una pérdida económica de R $\$ 342.473,00$ en 2015, cuando aún no existía la práctica de auditoría in situ, frente al valor de R\$114.157, en 2018, tras la acción de conferencias en el quirófano. Conclusión: Se logró un beneficio económico con la práctica de la auditoría in situ, con una disminución del 66,6\% en la cantidad de materiales y medicamentos excluidos por la auditoría, debido al aumento en la calidad de las notas en las historias clínicas.

Palabras clave: Enfermería de quirófano. Registros de hospitales. Costos y análisis de costo. Organización y administración.

'Enfermeira pela Faculdade Israelita de Ciências da Saúde Albert Einstein (FICSAE) - São Paulo (SP), Brasil.

2Doutora em Enfermagem pela Escola de Enfermagem da Universidade de São Paulo. Professora dos cursos de graduação e pós-graduação da FICSAE - São Paulo (SP), Brasil.

*Autora correspondente: prof.rachelcarvalho@gmail.com

Recebido: 09/09/2020 - Aprovado: 08/01/2021

https://doi.org/10.5327/Z1414-4425202100020004 


\section{INTRODUÇÃo}

Em meio a um cenário empresarial em que a economia em saúde vem ganhando papel extremamente relevante, evidencia-se que a elevação dos custos tem se constituído objeto de atenção por parte dos gestores hospitalares e das fontes pagadoras da assistência. As instituições hospitalares investem na auditoria de contas visando à adequada remuneração do atendimento prestado e ao faturamento das contas de maneira fiel ao que foi utilizado pelo paciente ${ }^{1}$.

No contexto das instituições hospitalares, o centro cirúrgico (CC) é um dos setores que mais utiliza recursos materiais, o que gera consumo elevado e, por conseguinte, torna esse espaço grande produtor de desperdícios. Portanto, esse setor representa um grande desafio para a administração de gastos excessivos ${ }^{2,3}$. Esse desperdício pode acarretar, ainda, produção ineficaz de processos, procedimentos e/ou serviços destinados à assistência ${ }^{4}$.

Os processos de auditoria contábil no CC têm a finalidade de conferir procedimentos realizados, materiais utilizados na anestesia e na cirurgia, medicações administradas e exames, em comparação com a conta hospitalar, para envio às fontes pagadoras ${ }^{4}$.

Seguindo uma tendência mundial de diminuir custos hospitalares por meio de novos modelos de gestão de custos ${ }^{5}$, o hospital-sede do estudo realiza uma pré-análise dos registros hospitalares intraoperatórios, in loco, dentro das salas operatórias (SO). Essa prática ocorre durante o procedimento cirúrgico e ao seu término. Posteriormente, os enfermeiros auditores conferem todos os itens das contas hospitalares relativas ao paciente, além do respectivo prontuário clínico, a fim de realizar correções munidos de um olhar muito mais amplo, pois estiveram presentes no momento do ato anestésico-cirúrgico. Essas práticas também possibilitam o desenvolvimento de uma atividade educativa constante com toda equipe que presta assistência direta ao paciente e é responsável pelo preenchimento dos formulários institucionais.

Tais correções, ajustes positivos (inclusões de materiais/ equipamentos) ou negativos (exclusões de materiais/equipamentos), fundamentam a cobrança dos procedimentos e evitam a ocorrência de glosas. Define-se glosa como o cancelamento ou a recusa parcial ou total de orçamento, conta, verba, por serem considerados ilegais ou indevidos, referentes aos itens que os auditores das operadoras de saúde não consideram cabíveis no pagamento ${ }^{6}$.
Os processos assistenciais geram receita por meio dos registros das ações realizadas. Destaca-se que parte significativa do pagamento de materiais, medicamentos e procedimentos vincula-se aos registros dos profissionais de saúde que, geralmente, são inconsistentes, ilegíveis e subjetivos, ocasionando glosas de itens do faturamento, erros em cobranças e, consequentemente, perda de receita ${ }^{7}$. Um estudo realizado no Rio Grande do Sul apontou que $90 \%$ das glosas ocorrem em razão do preenchimento inadequado e/ou incompleto dos dados do prontuário: evolução clínica e de enfermagem, prescrição médica, relatório de enfermagem, descrição cirúrgica e ficha de anestesia. Entre os 218 prontuários analisados pelos autores, $77 \%$ careciam de preenchimento adequado 8 .

Assim, o preenchimento inadequado repercute na sustentação econômica e no ato de controladoria do hospital, pois reverte-se em pagamentos indevidos referentes à conta hospitalar'. Na visão contábil e financeira da instituição, a auditoria contribui para amenizar a geração de gastos desnecessários, porque é uma ferramenta que propicia a identificação dos focos de geração de custos e propõe medidas de intervenção com futuros critérios de avaliação ${ }^{10}$.

A fim de dimensionar o tamanho do prejuízo financeiro que a ausência de registros em prontuário pode trazer, além da inexistência dessa prática de conferências in loco, foram elencados os materiais que, em razão da ausência de registro em prontuário, podem representar ofensa ao faturamento das contas e à sustentabilidade financeira da instituição.

\section{OBJETIVOS}

Elencar quais são os materiais de maior impacto financeiro no CC de uma instituição hospitalar privada de São Paulo;

Estimar a perda de receita anual do CC por ausência de registros das ações realizadas pela equipe assistencial.

\section{MÉTODO}

Estudo retrospectivo, baseado em documentação primária, com análise quantitativa dos dados. O estudo foi realizado em um hospital geral da rede privada, de porte extra, com cerca de 720 leitos ativos, localizado na Zona Sul de São Paulo, que conta com dois CC. Um deles possui 14 SO e 10 leitos de Recuperação Pós-Anestésica (RPA), e o outro tem $21 \mathrm{SO}$ e 36 leitos de RPA, onde são realizados cerca de $3 \mathrm{mil}$ procedimentos anestésico-cirúrgicos por mês. 
Foram utilizados resultados do banco de dados e planilhas setoriais do CC, contabilizados para garantia da receita, tomando-se como base os registros dos anos de 2015 e 2018. Em 2015, na instituição-sede da pesquisa, houve o início da análise de dados sobre as perdas financeiras decorrentes de ausência de evidências em prontuário. Os resultados de tais análises deram origem a um projeto educativo e de mudança na cultura das cobranças para a equipe de assistência direta ao paciente. No ano de 2018, já havia na instituição um setor responsável pela atividade de conferências in loco e pelo levantamento de dados financeiros. Por esses motivos, os anos 2015 e 2018 foram escolhidos para o levantamento deste estudo.

O instrumento de coleta de dados foi utilizado na forma de planilha, na qual foram inseridas as informações acerca dos materiais com maior volume de dispensação pela farmácia e maior porcentagem de exclusão pela auditoria interna do hospital, referentes a cada um dos meses de 2015 e 2018, ou seja, de $1^{\circ}$ de janeiro a 31 de dezembro de 2015 e de $1^{\circ}$ de janeiro a 31 de dezembro de 2018. Ressalta-se que a planilha foi construída com uma profissional estatística, mestre em Matemática e Estatística.

Os dados foram coletados entre os meses de maio e agosto de 2019, após aprovação do projeto de pesquisa pela Comissão Científica, bem como após autorização da gerência do CC e do gerente do setor de Garantia da Receita da instituição-sede do estudo. Em se tratando de uma pesquisa de custos, que não envolve seres humanos, o projeto não necessitou de aprovação do Comitê de Ética em Pesquisa.

Os dados coletados foram transferidos para uma planilha de Excel, na qual sofreram tratamento quantitativo, segundo orientação estatística, e estão apresentados em números absolutos e percentuais na forma de tabela e gráficos/figuras.
Foram analisados os bancos de dados de dispensação dos materiais e medicamentos pela farmácia e subtraídos aos faturados pela auditoria, representando, assim, a quantidade de perda financeira de cada item. Foi considerada a variação percentual dos itens dispensados, utilizados e excluídos pela auditoria por falta de evidência, bem como os valores financeiros embasados no preço de custo de cada item, preestabelecido pela instituição.

\section{RESULTADOS}

Os resultados são apresentados em consonância com os objetivos propostos para este estudo, ou seja, o benefício financeiro que a auditoria in loco trouxe para o CC desde o início desta atividade, em 2015. Para a análise dos dados, consideraram-se materiais com maior volume de dispensação pela farmácia e maior percentual de exclusão pela auditoria interna do hospital, por conta da ausência de evidência em prontuário (Tabela 1 e Figura 1).

Quanto ao impacto que os desperdícios dos artigos causaram para a saúde financeira da instituição, foi realizado o cálculo por intermédio do preço de custo dos materiais dispensados pela farmácia e utilizados nos procedimentos cirúrgicos. A moeda utilizada para o cálculo foi o real (R\$).

No ano de 2015, quando ainda não havia a atividade de auditoria in loco, foram identificadas 54.441 unidades excluídas por falta de evidência médica e de enfermagem em prontuário. Três anos após o início das atividades, a avaliação do mesmo banco de dados apresentou declínio de $66,6 \%$, representado pelas 18.147 unidades excluídas em 2018. A soma dos materiais representou custo efetivo financeiro para a instituição de R\$342.473,00 em 2015, comparada a R\$114.157,00 em 2018.

Tabela 1. Materiais e medicamentos dispensados pela farmácia, utilizados e excluídos pela auditoria interna por ausência de evidência de uso em prontuário, no ano de 2018.

\begin{tabular}{|l|c|c|c|}
\hline Materiais/medicamentos & Dispensados & Excluídos por falta de evidência & $\%$ \\
\hline Sevorane ${ }^{\circledast}$ frasco $250 \mathrm{~mL}$ & 55.673 & 10.528 & 18,9 \\
\hline Plasma Lyte $^{\circledast} 500 \mathrm{~mL}$ & 7.518 & 1.157 & 15,3 \\
\hline Manta térmica superior & 8.181 & 623 & 7,6 \\
\hline Sensor monitor bispectral & 10.887 & 619 & 5,6 \\
\hline Máscara de anestesia adulto & 23.199 & 1.069 & 4,6 \\
\hline Caneta para bisturi elétrico & 21.195 & 744 & 3,5 \\
\hline Perneira Sequel ${ }^{\circledR}$ média & 5.591 & 137 & 2,4 \\
\hline Placa para bisturi elétrico & 23.395 & 565 & 2,4 \\
\hline
\end{tabular}




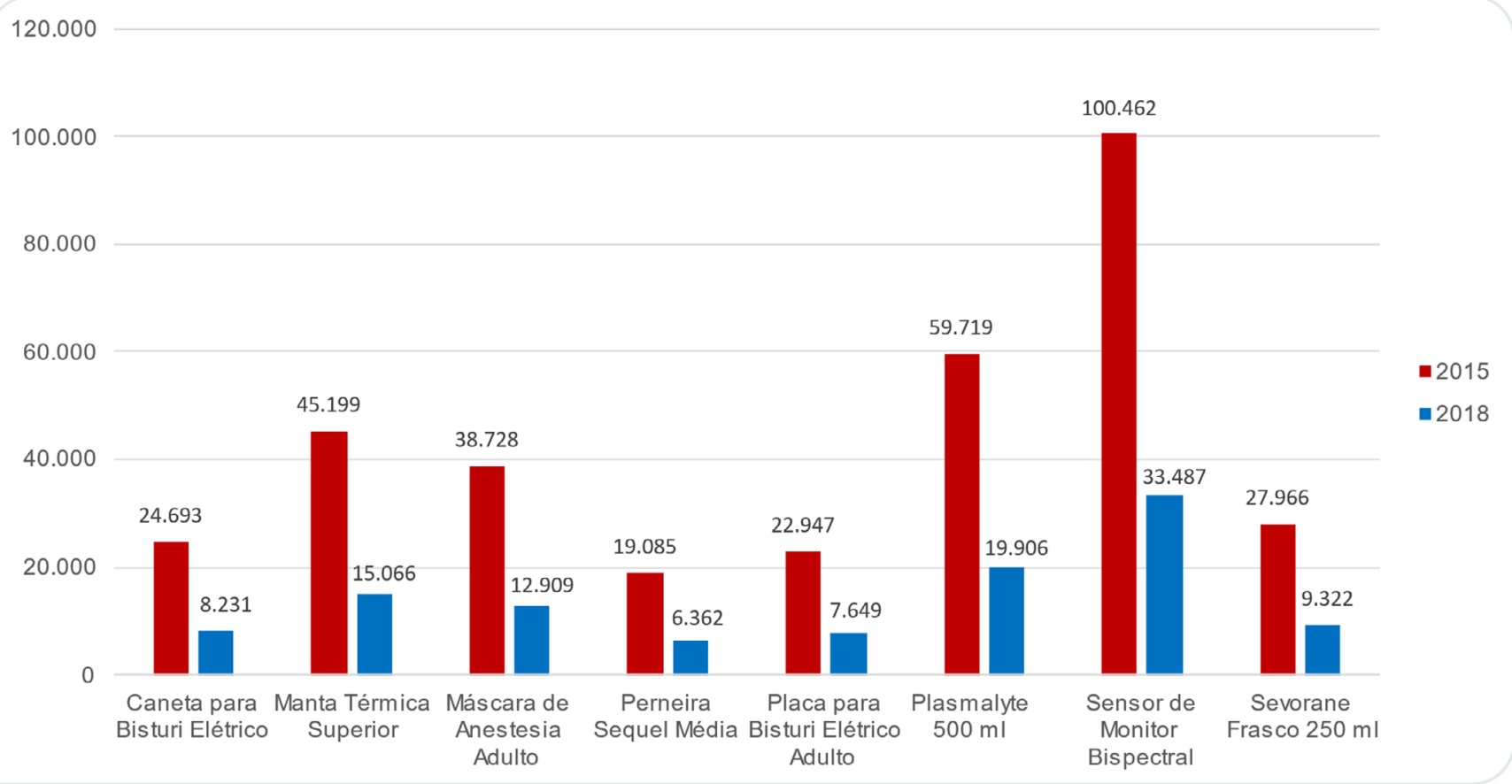

Figura 1. Perda de receita anual, por material, após auditoria interna, em razão da ausência de evidências em prontuário. Comparativo entre 2015 e 2018. Valores expressos em reais (R\$).

A variação de perda financeira quando não havia nenhum tipo de ação local foi de R\$ $100.462,00$ a R\$ $19.085,00$ ao longo do ano, comparada a um valor muito inferior, variando de $\mathrm{R} \$ 33.487,00$ a $\mathrm{R} \$ \mathbf{6 . 3 6 2 , 0 0}$ após a implementação das ações nas SO.

O mês que representou maior custo por ausência de evidências foi janeiro de 2015, quando os itens avaliados somaram o valor de R $\$ 47.779,00$. Em abril de 2015, observou-se queda nos valores, em razão do início de ações localizadas voltadas para educação e mudança da cultura de desperdício de materiais da equipe médica e assistencial. Os meses de menor perda financeira demonstram-se a partir de abril de 2018, quando a instituição já havia consolidado a ação e, principalmente, a interação da equipe assistencial com a equipe de auditoria in loco. O percentual de melhoria das anotações em prontuário chegou a $86,1 \%$ quando comparado ao pior mês sem auditoria in loco e ao melhor mês com a presença de ações concorrentes nas SO.

Desde a implantação da prática de auditoria in loco até o período atual, houve diminuição das ausências de evidências nas anotações de enfermagem e médica no prontuário do paciente, o que também proporcionou redução do intervalo de tempo entre a alta hospitalar e o encaminhamento da fatura à fonte pagadora, especialmente às operadoras de saúde.

\section{DISCUSSÃO}

A prática das enfermeiras auditoras faz parte de um processo educativo, e, embora o conhecimento sobre essa atividade ainda possa ser considerado recente, tem condições de contribuir de maneira significativa para a profissão ao criar circunstâncias para o gerenciamento de uma assistência de enfermagem de qualidade $^{11}$. A função da auditoria deve ir além de questões puramente financeiras, pois há também grande potencial de mudança de cultura de desperdício de materiais e anotações falhas.

O sensor de monitor bispectral foi o item que se demonstrou maior ofensor financeiro no ano de 2015. O índice bispectral é um parâmetro multifatorial derivado do eletroencefalograma, que permite a monitorização do componente hipnótico da anestesia ${ }^{12}$. Alguns estudos demonstram que a adequada monitoração da profundidade anestésica poderia reduzir a administração excessiva de agentes anestésicos, diminuir o tempo da recuperação anestésica, náuseas e vômitos, cefaleia e disfunções cognitivas ${ }^{13}$, fatores que também beneficiam a gestão de custos do CC. O valor elevado do sensor, unido à ausência de evidências do seu uso, fê-lo atingir um valor anual de perda de $\mathrm{R} \$ 100.462,00$. Esse item, que dependente exclusivamente de anotações médicas para o faturamento correto, caiu para $\mathrm{R} \$ 33.487,00$ após ações 
de conscientização do uso correto, anotação dos parâmetros em ficha anestésica e treinamento para posicionamento adequado, evitando desperdícios e indicação para cirurgias com duração superior a duas horas.

Outro item com alto valor percentual de exclusão foi o Sevorane ${ }^{\circledR}$, em frasco de $250 \mathrm{~mL}$. É um anestésico geral inalatório, de uso hospitalar, cuja administração tem sido associada à indução anestésica com perda de consciência rápida e suave, bem como à rápida recuperação após descontinuação da anestesia. É amplamente utilizado, pois abrange pacientes adultos e pediátricos e possui restrição apenas para casos de hipertermia maligna ou sensibilidade conhecida ${ }^{14}$. Por se tratar de um agente inalatório, a dimensão do seu uso em cada procedimento cirúrgico tornou-se um processo de difícil controle, causando uma perda anual de $\mathrm{R} \$ 27.966,00$. A ação de melhoria para esse indicador foi realizar um acordo comercial com as fontes pagadoras em que, para cada hora de uso do Sevorane ${ }^{\circledR}$ em SO, seria cobrado o valor equivalente a $30 \mathrm{~mL}$ do medicamento. Também foram realizadas orientações para todo o corpo médico de anestesiologistas sobre a nova forma de cobrança do medicamento. O processo de melhoria reduziu o custo para $\mathrm{R} \$ 9.322,00$, assumindo uma economia de mais de $\mathrm{R} \$ 18.000,00$ por ano.
Entre os materiais elencados pelo estudo, a manta térmica superior também representou grande ofensa financeira. Ela é utilizada em razão da diminuição da temperatura corporal que ocorre imediatamente após a indução de anestesia geral ou regional, decorrente da redistribuição de calor do compartimento central para o periférico. Nas intervenções cirúrgicas em que há exposição de cavidades corporais, a perda de calor é ainda maior, tornando ainda mais necessário seu uso com fluxo de ar aquecido ${ }^{15}$. Seu valor percentual de perda foi reduzido de 12,9 para $1,1 \%$ após ações educativas visando à qualidade dos registros dos enfermeiros e técnicos que atuam como circulantes de sala.

A atuação dos enfermeiros auditores é desconhecida por grande parte dos profissionais de enfermagem. Pesquisas mostram que a auditoria de enfermagem é relacionada basicamente aos custos hospitalares, à conferência e ao controle do prontuário médico ${ }^{6}$. Contudo, ao passo que esse profissional se aproxima da equipe de enfermagem assistencial e médica, eles conhecem suas funções e percebem nelas uma fonte de influências educativas e de aprimoramento na qualidade da assistência prestada ao paciente ${ }^{16}$.

Essa afirmação se confirma com os dados da Figura 2, que demonstra valores altos de perda financeira quando

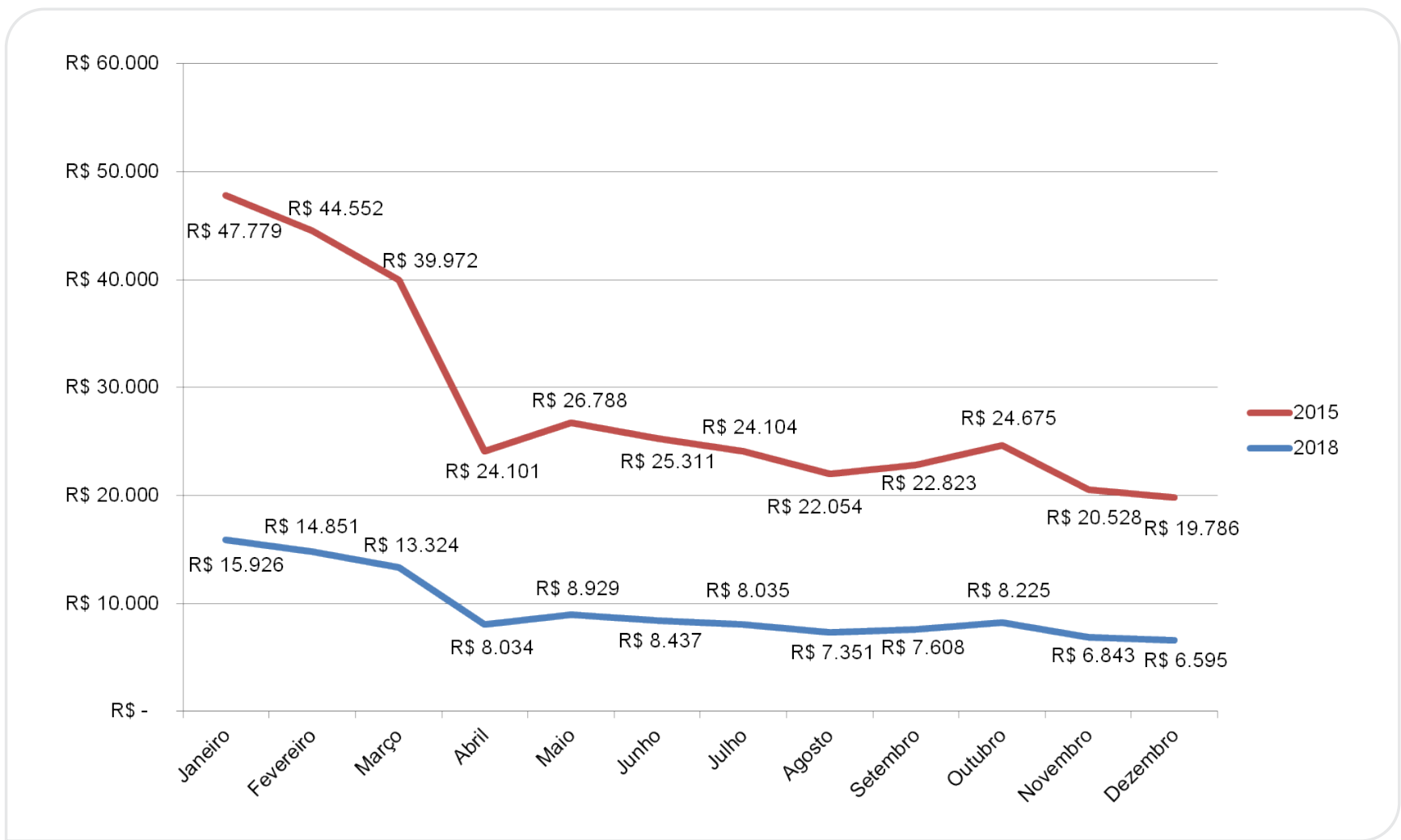

Figura 2. Custo mensal da ausência de evidências em prontuário. Mudança da qualidade das anotações entre os anos de $2015 \mathrm{e}$ 2018. Valores expressos em reais ( $R \$)$. 
não havia a auditoria in loco ( $\mathrm{R} \$ 100.462,00$ a $\mathrm{R} \$ 19.085,00) \mathrm{e}$ valores muito inferiores após a ação e fusão das duas equipes ( $\mathrm{R} \$ 33.487,00$ a $\mathrm{R} \$ 6.362,00$ ), quando todos puderam compreender a função real da auditoria, a importância da ação para a rentabilidade financeira da instituição e o impacto que a cultura do desperdício poderia trazer a longo prazo. Pensando nesses pontos é que a sensibilização dos profissionais torna-se fundamental, visando a uma reformulação no modo de se fazer auditoria.

Outro aspecto a ser observado refere-se à sustentabilidade das instituições de saúde, que precisam desenvolver-se economicamente por meio do gerenciamento eficiente de seus inúmeros processos. No Brasil ainda são poucas as investigações científicas sobre os custos da assistência de enfermagem, embora a atuação da equipe no controle de custos hospitalares contribua para a eficiência das instituições. O desconhecimento da relação entre o custo de procedimentos e a produtividade dos serviços favorece o desperdício e dificulta o surgimento de inúmeras possibilidades de aprimoramento da gestão na enfermagem ${ }^{17}$.

A falta de controle gerencial tem sido apontada como causa de ineficiência dos serviços de saúde, tanto pública como privada, sendo imprescindível a adoção de estratégias que auxiliem na descoberta mais rápida de desperdícios e falhas que aumentam os gastos nas organizações ${ }^{1}$. Tais falhas retratam alto custo para a instituição, representado, neste estudo, pelo valor de R $\$ 342.473,00$ por ano. Se a oportunidade de melhoria não tivesse sido identificada e esse valor se perdurasse até o ano de 2018, esse período traduziria uma perda financeira estimada em R\$1.027.419,00 para a instituição.

Nesse sentido, a implantação de auditoria in loco pode contribuir e endossar parâmetros de qualidade, podendo ser percebida como uma forma eficaz de gerenciamento de custos, por meio não apenas dos registros no prontuário de pacientes, mas também pela observação cotidiana da prática e pelo apoio às ações da equipe.

A incorporação de vários profissionais na busca do objetivo de redução de custos transformou a meta financeira em um compromisso para todos os colaboradores envolvidos $^{18}$.

A avaliação da efetividade e economicidade das ações em saúde tem na auditoria uma ferramenta eficaz de gestão, e esta, por sua vez, tem condições de contribuir para o complexo planejamento dos serviços de saúde. Pode instrumentalizar a equipe de enfermagem e reforçar a importância do enfermeiro auditor nesse processo por meio de ações e orientações, além da confecção de instrumentos que favoreçam os registros assistenciais ${ }^{19}$.

\section{CONCLUSÃO}

A realização do presente estudo, por meio da análise quantitativa do banco de dados, de planilhas setoriais do CC e de garantia da receita dos anos de 2015 e 2018, permitiu concluir que os materiais que causaram maior impacto financeiro para a instituição foram Sevorane ${ }^{\circledR}$, frasco de $250 \mathrm{~mL}$ (18,9\%), Plasma Lyte ${ }^{\circledR} 500 \mathrm{~mL}(15,3 \%)$, manta térmica superior $(7,6 \%)$, sensor do monitor bispectral $(5,6 \%)$, máscara de anestesia adulto $(4,6 \%)$, caneta para bisturi elétrico $(3,5 \%)$, perneira Sequel ${ }^{\circledR}$ média $(2,4 \%)$ e placa para bisturi elétrico adulto $(2,4 \%)$. Os materiais representaram perda financeira de $\mathrm{R} \$ 342.473,00$ no ano de 2015 , quando não havia a prática de auditoria in loco, comparada ao valor de $\mathrm{R} \$ 114.157,00$ no ano de 2018, após inserção de conferências em $S O$.

A experiência da evolução do modelo de auditoria retrospectiva para a in loco se mostrou positiva, pois foi possível perceber os benefícios financeiros obtidos em apenas três anos de atuação, além de um ganho intangível de mudança de cultura na postura dos profissionais assistenciais no que diz respeito à qualidade de registros em prontuário.

\section{Benefícios para enfermagem}

Adotando um pensamento crítico e reflexivo, é possível implementar novas formas de pensar sobre a auditoria de enfermagem no contexto hospitalar, assumindo o papel de educador diário no $\mathrm{CC}$ e interagindo com os profissionais e capacitando-os para as melhorias necessárias.

O trabalho das enfermeiras in loco estreita as relações com os demais profissionais, auxiliando, inclusive, na visão destes sobre a auditoria, não como um exercício fiscalizatório, mas assessório e educativo às boas práticas da enfermagem.

\section{Limitações do estudo}

Esta pesquisa foi realizada tomando-se como base materiais e equipamentos dispensados somente em SO. Pretende-se realizar novo estudo que englobe todo o bloco cirúrgico, incluindo itens dispensados na recuperação anestésica e no centro de material e esterilização. 


\section{REFERÊNCIAS}

1. Castilho V, Castro LC, Couto AT, Maia FOM, Sasaki NY, Nomura $\mathrm{FH}$, et al. Levantamento das principais fontes de desperdício de unidades assistenciais de um hospital universitário. Rev Esc Enferm USP. 2011;45(n. esp.):1613-20. https://doi.org/10.1590/ S0080-62342011000700012

2. Castro LC. O custo de desperdício de materiais de consumo em um centro cirúrgico [tese]. São Paulo: Escola de Enfermagem, Universidade de São Paulo; 2012.

3. Silva MJN, Ribeiro AL. Gestão em centro cirúrgico: identificação de desperdícios. Rev SOBECC. 2016;21(2):82-9. https://doi.org/10.5327/ Z1414-4425201600020004

4. Limoeiro ACO. Processos de auditoria no bloco cirúrgico. In: Carvalho $\mathrm{R}$, editor. Enfermagem em centro cirúrgico e recuperação anestésica. Manuais de especialização. Barueri: Manole; 2015. p. 282-9.

5. Falk JA. Gestão de custos para hospitais: conceitos, metodologias e aplicações. São Paulo: Atlas; 2011.

6. Motta ALC. Auditoria de enfermagem nos hospitais e operadoras de planos de saúde. 6a ed. São Paulo: látria; 2013. p. 166-9.

7. Guerrer GFF, Lima AFC, Castilho V. Estudo da auditoria de contas em um hospital de ensino. Rev Bras Enferm. 2015;68(3):358-63. https:// doi.org/10.1590/0034-7167.2015680306i

8. Lana LD, Ferraza CAC, Quadros JN, Birrer JA. Auditoria em organizações prestadoras de serviço: uma estratégia de gestão econômica. RAS. 2012;14(56):103-8.

9. Scarparo AF, Ferraz CA. Auditoria em enfermagem: identificando sua concepção e métodos. Rev Bras Enferm. 2008;61(3):302-5. https:// doi.org/10.1590/S0034-71672008000300004

10. Bessera EP, Alves MDS. Pesquisa sobre auditoria em serviços de saúde. Rev Adm Saúde. 2009;11(44):143-8.
11. Pinto KA, Melo CMM. A prática da enfermeira em auditoria em saúde. Rev Esc Enferm USP. 2010;44(3):671-8. https://doi.org/10.1590/ S0080-62342010000300017

12. Duarte LTD, Saraiva RA. Quando o índice bispectral (BIS) pode fornecer valores espúrios. Rev Bras Anestesiol. 2009;59(1):99-109. https:// doi.org/10.1590/S0034-70942009000100013

13. Nunes RR, Fonseca NM, Simões CM, Rosa DM, Silva ED, Cavalcante SL, et al. Consenso brasileiro sobre monitoração da profundidade anestésica. RevBras Anestesiol [lnternet]. 2015 [acessado em 10 nov. 2019];65(6):42736. Disponível em: http://www.scielo.br/pdf/rba/v65n6/pt_0034-7094rba-65-06-00427.pdf. https://doi.org/10.1016/j.bjan.2015.10.001

14. Sevorane [bula]. Buenos Aires: Abbott Laboratories Argentina AS; 2017.

15. Bernardis RCG, Silva MP, Gozzani JL, Pagnocca ML, Mathias LAST. Uso da manta térmica na prevenção da hipotermia intraoperatória. Rev Assoc Med Bras. 2009;55(4):421-6. https://doi.org/10.1590/ S0104-42302009000400017

16. Pereira PM, Petry P, Porto AR, Thofehrn MB. A importância do enfermeiro auditor na qualificação da assistência profissional. Rev Enferm UFPE OnLine [Internet]. 2010 [acessado em 28 out. 2019];2(4):504-9. Disponível em: https://pdfs.semanticscholar.org/ a6e5/e18a3d90fc62f552a9e24fa319fc7d4bc67a.pdf. https://doi. org/10.5205/reuol.644-7078-1-LE.0402201008

17. Albano TC, Freitas JB. Participação efetiva do enfermeiro no planejamento: foco nos custos. Rev Bras Enferm. 2013;66(3):3727. https://doi.org/10.1590/S0034-71672013000300011

18. Richa AC, Guimarães SM, Cardoso TV. Gestão por padronização de processos: a percepção dos enfermeiros de centro cirúrgico. Rev SOBECC. 2014;19(1):3-10. https://doi.org/10.4322/sobecc.2014.003

19. Menezes JGBRL, Bucchi SM. Auditoria em enfermagem: um instrumento para assistência de qualidade. Rev Enferm UNISA. 2011;12(1):68-72. 